\title{
Plasma-Wall Interactions in the Presence of Plasma Fluctuations - Interpretation of Line Emission from Sputtered Tungsten in PSI-2
}

\author{
D. Reiser, ${ }^{1}$ D. Borodin, ${ }^{1}$ S. Brezinsek, ${ }^{1}$ A.A. Eksaeva, ${ }^{1,2}$ A. Kirschner, ${ }^{1}$ A. Kreter, ${ }^{1}$ \\ J. Romazanov, ${ }^{1}$ and T. Schlummer ${ }^{1}$ \\ ${ }^{1}$ Forschungszentrum Jülich GmbH, Institut für Energie- und Klimaforschung - Plasmaphysik, \\ Partner of the Trilateral Euregio Cluster (TEC), 52425 Jülich, Germany \\ ${ }^{2}$ National Research Nuclear University MEPhl, 31, Kashirskoe sh., 115409, Moscow, Russia
}

\begin{abstract}
The analysis in this work essentially addresses the general question to what extent the temporal average of a particular quantity which is a highly non-linear function of fluctuating quantities can be approximated by using the averages of the fluctuating quantities for its evaluation. The concrete case considered is the line emission intensity from sputtered impurities being a function of fluctuating electron density and temperature in a plasma beam of the PSI-2 device. A threedimensional fluid model is employed to study the impact of plasma fluctuations on the distribution of particles and line emission in PSI-2 discharges and its interpretation in long-term measurements. In the model presented the solution of a vorticity equation to obtain a self-consistent electric field is avoided and a synthetic turbulent velocity field is included instead. This approach is based on a Langevin model including advection and allows numerically efficient parameter scans by controlling amplitude, correlation length and correlation time of plasma fluctuations known from extended 3D simulations and/or experiment. The synthetic turbulence model considered is an extension of established stochastic models used for studies of passive scalar advection and therefore, it is described in detail in a general framework. Numerical examples of PSI-2 applications show that a double log-normal probability density function for the electrons and impurity ions is likely to occur and that this supports the conclusion that very high levels of intermittency are required to find a significant impact on the experimental evaluation method which is based on temporal averages only. Consequently, for typical PSI-2 experiments the method of evaluation based on averaged plasma parameters is justified.
\end{abstract}

\section{INTRODUCTION}

In studies of Plasma-Wall Interaction (PWI) the experimental analysis of erosion processes of target materials such as tungsten, beryllium and iron relies on a variety of diagnostic tools determining the characteristics of the plasma (Langmuir probes, Thomson scattering, Laser Induced Fluorescence etc.) and the transport of eroded material (Optical Emission Spectroscopy, Quartz Microbalance etc.). The data obtained by these diagnostic means are used to infer information on the amount and the distribution of eroded materials and the underlying PWI processes. Due to technical constraints in most cases these data represent a temporal average over intervals of $\mathrm{ms}$ or even longer. On the other hand large amplitude plasma fluctuations of about $50 \%$ or even more on time scales of $\mu$ s are known to be present in many devices. This raises the question whether the use of averaged data in post-processing is justified to deduce the required information on impurity distribution and transport. Currently, dedicated tungsten (W) sputtering experiments in the linear plasma device PSI-2 [1;2] and numerical simulations using the ERO code [3-5] show particular discrepancies in line radiation intensities of sputtered neutral W [6]. Possible reasons for the discrepancies are missing model details like uncertainties in atomic data, non-Maxwellian features in the electron distribution function or plasma fluctuations not being resolved in the experiment and in the ERO simulations. To discuss the latter point the present paper considers a time-dependent three-dimensional fluid model used to mimic those $\mathrm{W}$ sputtering experiments in PSI-2. The aim of this numerical study, however, is not to improve the reconstruction of experimental results by a more refined numerical model, but to extract more qualitative results to clarify whether plasma fluctuations might be responsible for the above mentioned discrepancy. In this work the transport simulation of the main plasma includes small scale fluctuations and therefore, the sputtered $\mathrm{W}$ reflects this irregular temporal behaviour due to strong modulations in sputter yields and mean free paths. This point has been discussed marginally already in Ref. [9]. But due to the time-consuming numerics used there it was not pursued further. Therefore, an essential part of the present work is the inclusion of a synthetic turbulence in a simplified fluid description of the sputtering process at the target. This allows a very quick numerical scan through parameter regimes of interest. The synthetic turbulence model is implemented in the fluid simulations by generating a correlated velocity field perpendicular to the magnetic field. The resulting passive scalar model is based on empirical statistical data, i.e. correlation length, correlation time and fluctuation amplitude taken from extended 3D simulations and/or experiment. The numerical simplification obtained allows quick parameter scans for the scenarios of interest. Results for typical PSI-2 experimental conditions are shown with particular focus on the region of maximum line intensity, where the discrepancies between experiment and ERO reconstruction are most pronounced. The averages of fluctuating $\mathrm{W}$ density and line intensity are compared with the respective results based on using averaged background plasma 
profiles. A detailed statistical analysis is presented to estimate the range of validity of the approximative averaging procedure and to find out limits for the need of including fluctuations in the ERO modelling.

\section{OPTICAL SPECTROSCOPY VS ERO SIMULATION}

The linear plasma device PSI-2 provides an almost axisymmetric magnetically confined plasma column. The magnetic field strength is of the order of $0.1 \mathrm{~T}$ and for argon (Ar) as working gas - electron temperatures and particle densities are of the order of $T_{e} \sim 2-4 \mathrm{eV}$ and $n_{e} \sim 10^{18} \mathrm{~m}^{-3}$, respectively. In the above mentioned sputtering experiments $\mathrm{W}$ targets were exposed to Ar plasma flows. During the experiments with duration of about minutes the electron density, electron temperature and the $4009 \AA$ line emission intensity of neutral W are measured routinely. Afterwards the time-averaged plasma parameters are used as an input for the 3D Monte-Carlo code ERO [3-5]. This code is used for the numerical simulation of trace impurity transport in a given background plasma taking into account detailed plasma wall interaction processes. To a large extent the comparison of experimental results and ERO simulations gave good agreement. However, for certain operational regimes ("high" discharge power) the ERO code absolute values of line intensity differ by factors up to 5 -8. It has been speculated that this might be caused by the nonlinear dependence of transport, sputtering and photon emission coefficients on fluctuating plasma density and temperature. Detailed information on the experiments and the comparison with ERO results can be found in [6].

\section{SIMPLIFIED FLUID MODEL FOR LINE EMISSION PROFILES}

To conduct a detailed numerical simulation of plasma wall interaction and self-consistent plasma fluctuations in principle a model like the one used in the ERO code should be combined with a global or local plasma turbulence code, e. g. based on an electrostatic drift fluid model in a cylindrical geometry. Drift fluid models of this kind are widely used for theoretical studies of plasmas in linear devices [9-21] and have been proven successful in the analysis and interpretation of experimental findings. However, such a combined model usually suffers from unacceptable long computing times and requires detailed knowledge about sources for particles and heat, boundary conditions, vorticity damping etc., not easily obtained from experiment. Therefore, in the present work it is not intended to obtain an improved match between simulation and experiment, but rather a more qualitative picture is the goal to identify the range of possibilities for the impact of plasma fluctuations in the experiment and its consequences in evaluating time averaged quantities like line emission intensity. For this purpose, the most basic processes of $\mathrm{W}$ transport in Ar plasma are described by the following fluid model. Electron density $n_{e}$ and temperature $T_{e}$ of the plasma containing singly charged Ar ions end electrons evolve according to

$\frac{\partial n_{e}}{\partial t}+\mathbf{V}_{\perp} \cdot \nabla n_{e}=S_{n}-\nu_{n} n_{e}$

$\frac{\partial T_{e}}{\partial t}+\mathbf{V}_{\perp} \cdot \nabla T_{e}=S_{T}-\nu_{T} T_{e}$

The density $n_{\mathrm{W}}$ of sputtered material (neutral $\mathrm{W}$ atoms) is assumed to obey

$\frac{\partial n_{\mathrm{W}}}{\partial t}+\mathbf{V}_{\|} \cdot \nabla n_{\mathrm{W}}=-k_{i} n_{\mathrm{W}}$

From this model also the local intensity $I_{\mathrm{W}}=p n_{\mathrm{W}}$ of emitted light of sputtered material is obtained. Here $p=p\left(n_{e}, T_{e}\right)$ is the photon emission coefficient. The quantities $S_{n}$ and $S_{T}$ describe source terms for particles and heat, reflecting the hollow cathode configuration of PSI-2. The rate coefficients $\nu_{n}$ and $\nu_{T}$ describe loss mechanisms due to parallel transport along the magnetic field in the plasma column. The ionization rate of neutral $\mathrm{W}$ is denoted by $k_{i}$. The parallel velocity $\mathbf{V}_{\|}$is given by the energy of sputtered particles and the perpendicular velocity $\mathbf{V}_{\perp}=\mathbf{B} \times \nabla \phi / B^{2}$ represents the $E \times B$-velocity containing both a stationary axisymmetric piece responsible for rotation of the plasma column and a fluctuating piece due to fluctuating electric fields. The plasma parameters $n_{e}$ and $T_{e}$ are assumed to have negligible parallel variations, i.e. $\nabla_{\|} n_{e}=\nabla_{\|} T_{e}=0$. Altogether, this model describes a fluctuating parallel plasma flux towards a target, the sputtering of target material and its parallel motion through the plasma column. The same rates coefficients for ionization, sputter yield and photon emission as in the ERO-code are used in the numerical solution of Eqs. 1-3. The source terms and loss rates are chosen in a way to get close to experimental profiles and also by the use of estimates taken from preparative 3D simulations, similar to the studies conducted in Refs. [9-11]. The fluctations in $\mathbf{V}_{\perp}$ are determined by statistical parameters as described in the next section.

\section{SYNTHETIC TURBULENCE}

The concept of synthetic turbulence is widely used to generate turbulent fields, in particular velocity fields, in order to reconstruct observations or to model turbulent motion when the basic statistical characteristics are known. The derivations below are based on the work of A. Careta et al. reported in Ref. [7]. The particular new point here is that it is applied to inhomogeneous turbulence on a disk. To model the turbulent piece in the velocity field $\mathbf{V}_{\perp}$ a $2 \mathrm{D}$ stochastic Langevin equation for the electric potential $\phi$ is considered

$\tau \frac{\partial \phi}{\partial t}=-\phi+\epsilon Q(\zeta)$ 

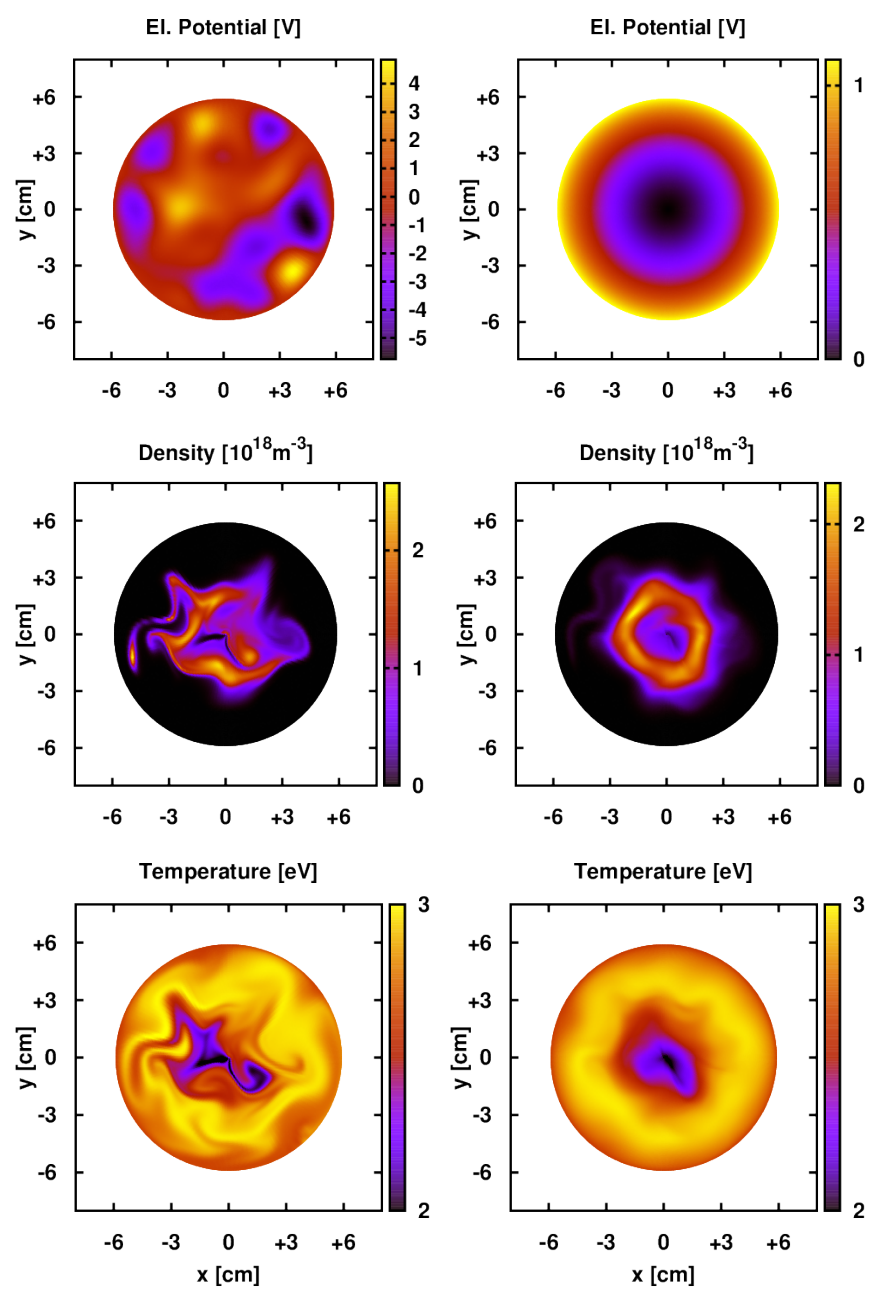

FIG. 1 Snapshots (left) and temporal averages (right) of electric potential (top), electron density (mid) and electron temperature (bottom) for a synthetic turbulence simulation using $\epsilon=49.0$ and other parameters as listed in Sec. V.

Here $Q$ is a spatial filter on a Gaussian white noise $\zeta$ whose correlation is

$\left\langle\zeta\left(\mathbf{r}_{1}, t_{1}\right) \zeta\left(\mathbf{r}_{2}, t_{2}\right)\right\rangle=\delta\left(\mathbf{r}_{1}-\mathbf{r}_{2}\right) \delta\left(t_{1}-t_{2}\right)$

The parameter $\epsilon$ is the amplitude of the noise and $\tau$ is the correlation time of the colored noise $\phi$. The filter operator $Q$ is assumed to be a functional of the perpendicular Laplacian $\nabla_{\perp}^{2}=\nabla^{2}-\nabla_{\|}^{2}$, where $\nabla_{\|}=\partial / \partial z$ and $z$ is the axial coordinate being aligned to the magnetic field. The fields $\phi$ and $\zeta$ are expanded using base functions $f_{m, n}$ given inside a circular domain $r \leq R, 0 \leq \theta \leq 2 \pi$ as

$\phi=\sum_{m, n} \phi_{m, n} f_{m, n} \quad, \quad \zeta=\sum_{m, n} \zeta_{m, n} f_{m, n}$

where

$f_{m, n}(r, \theta)=\frac{J_{m}\left(\nu_{m, n} r / R\right) e^{i m \theta}}{\sqrt{\pi} R J_{m+1}\left(\nu_{m, n}\right)}$

The $J_{m}$ are the Bessel functions of first kind and $\nu_{m, n}$ are their particular zeroes. Thus, the $f_{m, n}$ are eigenfunctions of $\nabla_{\perp}^{2}$, fulfilling the relation

$\nabla_{\perp}^{2} f_{m, n}=-\frac{\nu_{m, n}^{2}}{R^{2}} f_{m, n}$

and the boundary condition $f_{m, n}(R, \theta)=0$. The expansion leads to

$\tau \frac{\partial \phi_{m, n}}{\partial t}=-\phi_{m, n}+\epsilon Q_{m, n} \zeta_{m, n}$

which is an Ornstein-Uhlenbeck process for the expansion coefficients $\phi_{m, n}$. The solution is given by

$\phi_{m, n}(t+\Delta t)=\phi_{m, n}(t) \exp \left(-\frac{\Delta t}{\tau}\right)+\omega_{m, n}$

where

$\omega_{m, n}=\frac{\epsilon}{\tau} \int_{t}^{t+\Delta t} \exp \left(-\frac{t+\Delta t-t^{\prime}}{\tau}\right) Q_{m, n} \zeta_{m, n} d t^{\prime}$

is a Gaussian random number with zero mean and variance [8]

$\left\langle\left|\omega_{m, n}\right|^{2}\right\rangle=\sigma_{m, n}^{2}\left[1-\exp \left(-\frac{2 \Delta t}{\tau}\right)\right]$

The stationary probability density $p\left(\phi_{m, n}\right)$ is

$p\left(\phi_{m, n}\right)=\frac{1}{\sqrt{2 \pi \sigma^{2}}} \exp \left(-\frac{\left|\phi_{m, n}\right|^{2}}{2 \sigma_{m, n}^{2}}\right)$

This means that $\phi_{m, n}$ is Gaussian random number with zero mean and variance

$\sigma_{m, n}^{2}=\frac{\epsilon^{2} Q_{m, n}^{2}}{2 \tau}$

Finally one finds the discrete algorithm

$$
\begin{array}{r}
\phi_{m, n}(t+\Delta t)=\phi_{m, n}(t) \exp \left(-\frac{\Delta t}{\tau}\right) \\
+\alpha_{m, n} \sigma_{m, n} \sqrt{1-\exp \left(-\frac{2 \Delta t}{\tau}\right)}
\end{array}
$$

where $\alpha_{m, n}$ are Gaussian distributed random numbers with zero mean and variance 1 . Thus, in real space the complete solution at time $t+\Delta t$ can be written

$$
\begin{aligned}
\phi(r, \theta, t+ & \Delta t)=\sum_{m, n} \frac{J_{m}\left(\nu_{m, n} r / R\right) e^{i m \theta}}{\sqrt{\pi} R J_{m+1}\left(\nu_{m, n}\right)} \\
& \times\left(\phi_{m, n}(t) e^{-\Delta t / \tau}+\omega_{m, n}\right)
\end{aligned}
$$

Thus, together with the choice

$Q_{m, n}=e^{-\lambda^{2} \nu_{m, n}^{2} / R^{2}}$ 
the Eq.16 gives the numerical recipe to construct the temporal evolution of the correlated turbulent velocity field $\mathbf{V}_{\perp}$ by prescribing $\epsilon, \lambda$ and $\tau$. This extends the plasma evolution equations 1 and 2 by a model for passive scalar turbulence. Examples of snapshots and temporal averages for a simulation run with $\epsilon=49.0$ and other parameters as described in the next section are shown in Fig. 1.

\section{SIMULATION RESULTS}

Simulations based on the model and formulas described in the previous sections have been conducted for the following choice of parameters: parallel domain length $L_{z}=0.04 \mathrm{~m}$, radius of domain $R=0.06 \mathrm{~m}$, time step $\Delta t=10^{-8} \mathrm{~s}$, particle and heat source $S_{n}=10^{23} \mathrm{~m}^{-3} \mathrm{~s}^{-1}$, $S_{T}=3 \cdot 10^{4} \mathrm{eV} \mathrm{s}^{-1}$, loss rates $\nu_{n}=3 \cdot 10^{6} \mathrm{~s}^{-1}$ and $\nu_{T}=10^{6}$ $\mathrm{s}^{-1}$. Additionally a rotation frequency $\omega=2 \pi \cdot 10^{4} \mathrm{~s}^{-1}$ is taken into account adding a poloidal rotation velocity $\omega r$ to the velocity $\mathbf{V}_{\perp}$. The simulation runs cover a period of $1 \mathrm{~ms}$. The particular form of the hollow plasma source in PSI-2 is accounted for by multiplying the source terms $S_{n}$ and $S_{T}$ with a shape function $\exp \left[-4\left(r-r_{0}\right)^{2} / \Delta^{2}\right]$, where for particles $r_{0}=0.02 \mathrm{~m}$ and $\Delta=0.01 \mathrm{~m}$ has been chosen and for the heat source $r_{0}=0.04 \mathrm{~m}$ and $\Delta=0.1$ $\mathrm{m}$. To modify the strength of turbulence and to match the model with observations in experiments and more extended numerical simulations, like the ones described in Refs. [9-11], the control parameters for the synthetic turbulence have been chosen as $\tau=3 \cdot 10^{-5} \mathrm{~s}, \lambda=6 \mathrm{~mm}$ and $\epsilon=24.5,49.0,98.0$ and 196.0. A 2D profile of line emission intensity averaged over the entire simulation time is shown in Fig.2, left, for the case $\epsilon=49.0$. The right
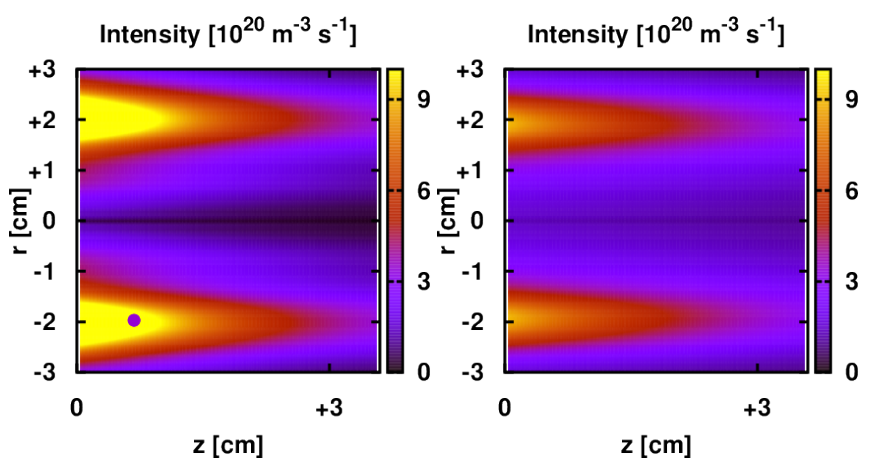

FIG. 2 Patterns of line intensity of $W_{0}$ in a turbulent $\mathrm{Ar}$ plasma with $\epsilon=49.0$ averaged over $1 \mathrm{~ms}$ (left) and obtained by using the corresponding temporal averages as stationary $\mathrm{Ar}$ plasma background (right). The small purple dot at $r=0.02 \mathrm{~m}$ and $z=7 \cdot 10^{-3} \mathrm{~m}$ in the left figure marks the location for sampling of the Probability Density Functions shown in Figs. 3 and 4 .

figure in Fig.2 shows the intensity profile for a continuation run, with background parameters $n_{e}$ and $T_{e}$ frozen to their averaged profiles evolved in the previous simulation including fluctuations which led to the result shown in the left figure. It can be seen that the profiles are al-
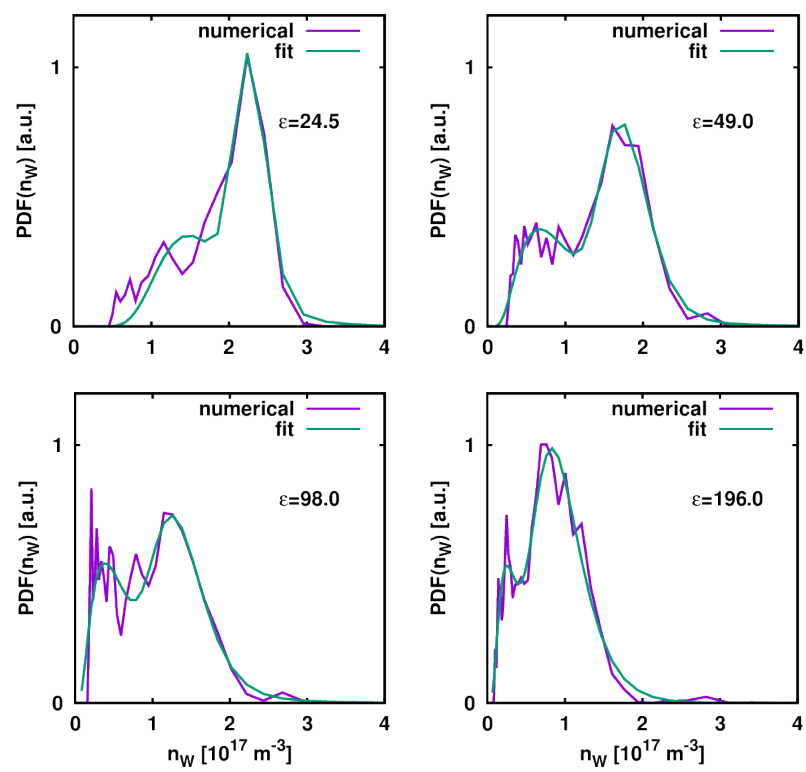

FIG. 3 PDF's of W density $n_{W}$ at $z=0.01 \mathrm{~m}$ and $r=0.02 \mathrm{~m}$ for varying amplitude $\epsilon$ of potential fluctuations. Shown are the numerically obtained results (purple) and the fit curves (green) according to Eq. 18
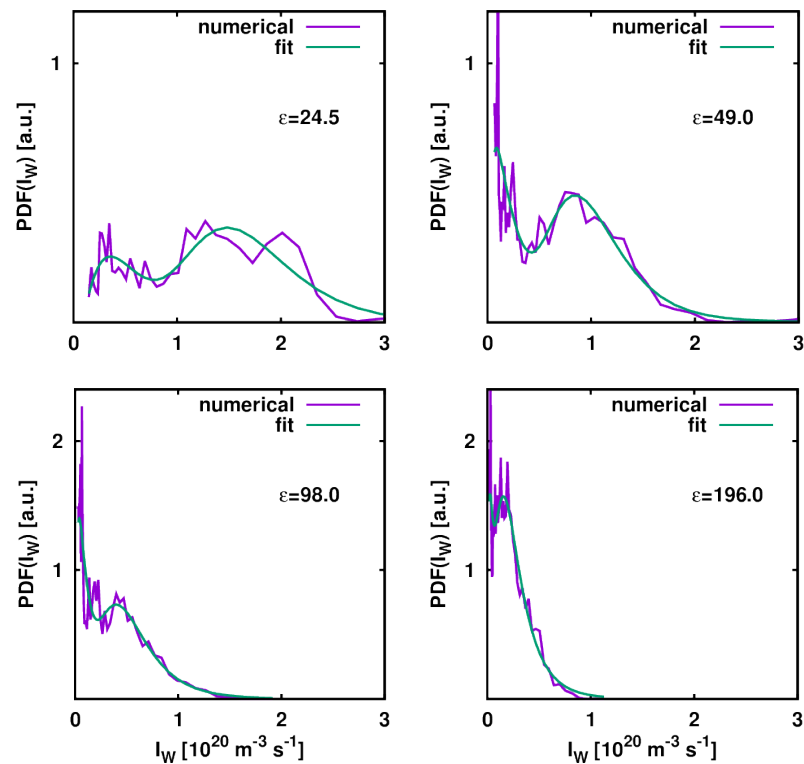

FIG. 4 PDF's of W line emission intensity $I_{W}$ at $z=0.01 \mathrm{~m}$ and $r=0.02 \mathrm{~m}$ for varying amplitude $\epsilon$ of potential fluctuations. Shown are the numerically obtained results (purple) and the fit curves (green) according to Eq. 18

most identical. Only a slight increase in the simulation including fluctuations can be found, but much less than a factor of 2. This is now further investigated by approximating the numerically found Probability Density Functions (PDF's) of the logarithmic quantities $\ln n_{W}$ and $\ln I_{W}$ using the fit-function $F$ defined by a weighted 
sum of two Gaussians

$F(Y)=\frac{A_{1} e^{-\frac{\left(Y-\mu_{1}\right)^{2}}{2 \sigma_{1}^{2}}}+A_{2} e^{-\frac{\left(Y-\mu_{2}\right)^{2}}{2 \sigma_{2}^{2}}}}{\sqrt{2 \pi}\left(A_{1} \sigma_{1}+A_{2} \sigma_{2}\right)}$

In Figs. 3 and 4 the PDF's of $\mathrm{W}$ density $n_{W}$ and line emission Intensity $I_{W}$ are shown sampled at a particular point at the maximum of the temporal average of line density (at $r=0.02 \mathrm{~m}$ and $z=7 \cdot 10^{-3} \mathrm{~m}$, marked in Fig. 2 left). It can be seen that the fit function $F$, which actually results in a double log-normal distribution for $n_{W}$ and $I_{W}$, gives a good approximation for the numerically obtained PDF's. Additionally it is to be mentioned (without illustrating figures), that a closer inspection of the numerical time evolution of the intensity $I_{W}$ proves that its fluctuations are predominantly determined by the boundary conditions at the target, i. e. by the fluctuating sputter yield. This is proportional to the electron density $n_{e}$ and its PDF is double log-normal too. The origin of the pronounced log-normal characteristics in the passive scalar dynamics is not yet fully understood. Based on our current state of knowledge the trajectories of electron density $n_{e}$ and radial velocity $v_{r}=\mathbf{e}_{r} \cdot \mathbf{V}_{\perp}$ as random variables in $n_{e}-v_{r}$-phase space oscillate around two attractors. But this kind of studies is just at the very beginning and has to be continued. However, for the present purposes the fact that the $\mathrm{W}$ distribution obeys log-normal statistics allows to draw some general conclusions. The mean value of a random quantity $X$ with $\ln X$ obeying a PDF given by Eq. 18 is found to be

$\bar{X}=\frac{A_{1} \sigma_{1} e^{\mu_{1}+\frac{\sigma_{1}^{2}}{2}}+A_{2} \sigma_{2} e^{\mu_{2}+\frac{\sigma_{2}^{2}}{2}}}{A_{1} \sigma_{1}+A_{2} \sigma_{2}}$

This means that the variances $\sigma_{1}^{2}$ and $\sigma_{2}^{2}$ of the underlying Gaussian processes introduced by the fluctuating velocity field can change $\bar{X}$ significantly. The limit of vanishing variances $\sigma_{1}, \sigma_{2} \rightarrow 0$ is found to be

$\bar{X}^{*}=\frac{A_{1} e^{\mu_{1}}+A_{2} e^{\mu_{2}}}{A_{1}+A_{2}}$

Indeed, if $X$ is identified with the $\mathrm{W}$ density or line emission the quantity $\bar{X}^{*}$ represents the average value in a frozen averaged plasma background shown in Fig. 2 right and being in the focus of this work. Therefore, it is reasonable to define an amplification factor $\gamma=\bar{X} / \bar{X}^{*}$ as a measure for the range of validity when using averaged background parameters for the approximative evaluation of line intensities as done in the ERO simulations discussed. The tables Tab. I and II list the fitting parameters found for the curves of Figs. 3 and 4 and the corresponding averages and amplification factors $\gamma$. The averaged density $\left\langle n_{W}\right\rangle$, and the averaged intensity $\left\langle I_{W}\right\rangle$ decrease with increasing turbulence amplitude $\epsilon$. This is due to the fact that the particles in the plasma beam are transported radially by the turbulence and with increasing turbulence the radial profiles of electron density become more and more flattened. Thus, the local electron density is reduced at the particular point considered here which can be regarded as an increase in effective radial diffusion. Because the $\mathrm{W}$ sputtering goes linearly with the electron density the impurity density and line intensity decrease too. In all examples shown the amplification factor $\gamma$ is close to 1. Also listed are the averages and amplification factors found by direct evaluation of the numerical time traces. The difference between the results from the PDF fit and the direct evaluation is always in the range of $10 \%$ or less, proving that Eq. 18 provides a good approximation. A possible increase of the amplification factor is illustrated by Fig. 5. It can be seen that if all other parameters are kept unchanged a value $\sigma_{1}>1.5$ is needed to obtain an amplification of $\gamma=3$. However, the log-normal statistics with such high values for $\sigma_{1}$ and/or $\sigma_{2}$ would also correspond to strongly intermittent plasma fluctuations in the plasma density $n_{e}$, i. e. irregular rare events of large amplitude. This is observed neither in the PSI-2 experiments nor in extended 3D simulations for the parameter ranges explored. Further work is needed to find possible paths to such highly intermittent regimes in PSI-2 operational regimes.

\begin{tabular}{|l|r|r|r|r|r|r|r|r|r|r|}
\hline$\epsilon$ & $A_{1}$ & $\mu_{1}$ & $\sigma_{1}$ & $A_{2}$ & $\mu_{2}$ & $\sigma_{2}$ & $\bar{n}_{W}$ & $\gamma$ & $\left\langle n_{W}\right\rangle$ & $\langle\gamma\rangle$ \\
\hline \hline 24.5 & 4435 & 39.6 & 0.32 & 4671 & 40.0 & 0.09 & 1.82 & 0.92 & 1.92 & 0.97 \\
\hline 49.0 & 4232 & 39.1 & 0.57 & 5213 & 39.7 & 0.17 & 1.30 & 0.93 & 1.46 & 0.97 \\
\hline 99.0 & 4701 & 38.7 & 0.70 & 4806 & 39.5 & 0.24 & 0.99 & 0.95 & 1.09 & 0.96 \\
\hline 196.0 & 2345 & 38.1 & 0.59 & 7055 & 39.1 & 0.34 & 0.80 & 0.98 & 0.86 & 0.97 \\
\hline
\end{tabular}

TABLE I Fit parameters for the PDF's of $n_{W}$ shown in Fig. 3 for varying turbulence amplitude $\epsilon$. The averages $\bar{n}_{W}$ are given in units of $10^{17} \mathrm{~m}^{-3}$. The averages $\bar{n}_{W}$ and amplification factors $\gamma$ are computed via Eqs. 19 and 20. The averages $\left\langle n_{W}\right\rangle$ and amplification factors $\langle\gamma\rangle$ are the numerically evaluated values.

\begin{tabular}{|l|l|l|l|l|l|l|l|l|l|l|}
\hline$\epsilon$ & $A_{1}$ & $\mu_{1}$ & $\sigma_{1}$ & $A_{2}$ & $\mu_{2}$ & $\sigma_{2}$ & $\bar{I}_{W}$ & $\gamma$ & $\left\langle I_{W}\right\rangle$ & $\langle\gamma\rangle$ \\
\hline
\end{tabular} \begin{tabular}{|l|l|l|l|l|l|l|l|l|l|l|}
\hline 24.5 & 5144 & 35.0 & 0.31 & 2464 & 34.0 & 0.69 & 12.61 & 0.94 & 13.18 & 1.04 \\
\hline
\end{tabular} \begin{tabular}{|l|l|l|l|l|l|l|l|l|l|l|}
\hline 49.0 & 4506 & 34.5 & 0.36 & 3358 & 33.2 & 1.06 & 6.37 & 0.96 & 7.73 & 1.10 \\
\hline 9
\end{tabular} \begin{tabular}{|l|l|l|l|l|l|l|l|l|l|l|l|l|}
\hline 99.0 & 4222 & 34.0 & 0.46 & 3631 & 32.6 & 1.13 & 3.93 & 1.03 & 4.37 & 1.13 \\
\hline 196.0 & 5517 & 33.2 & 0.61 & 2164 & 32.0 & 1.00 & 2.43 & 1.15 & 2.64 & 1.13 \\
\hline
\end{tabular} \begin{tabular}{|l|l|l|l|l|l|l|l|l|l|l|l|}
\hline 196.0 & 5517 & 33.2 & 0.61 & 2164 & 32.0 & 1.00 & 2.43 & 1.15 & 2.64 & 1.13 \\
\hline
\end{tabular}

TABLE II Fit parameters for the PDF's of $I_{W}$ shown in Fig. 4 for varying turbulence amplitude $\epsilon$. The averages $\bar{I}_{W}$ are given in units of $10^{20} \mathrm{~m}^{-3} \mathrm{~s}^{-1}$. The averages $\bar{I}_{W}$ and amplification factors $\gamma$ are computed via Eqs. 19 and 20. The averages $\left\langle I_{W}\right\rangle$ and amplification factors $\langle\gamma\rangle$ are the numerically evaluated values.

\section{CONCLUSION}

A reasonable choice of parameters for the passive scalar model proposed allows a good approximation of turbulent dynamics in PSI-2 experiments found experimen- 


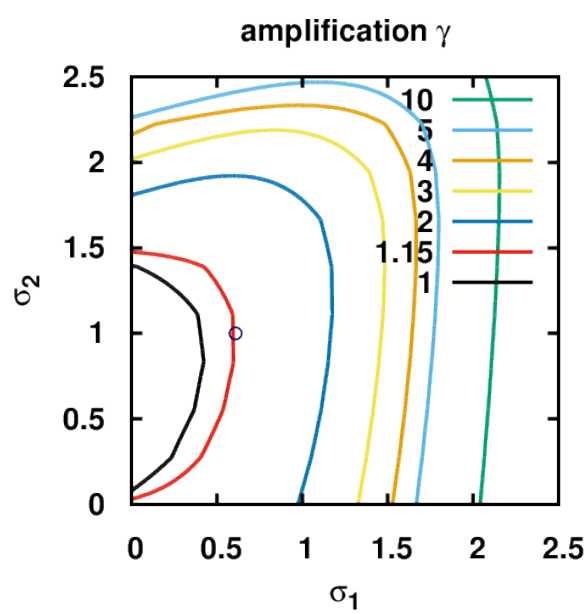

FIG. 5 Illustration of variation of amplification factor $\gamma$ with the variances $\sigma_{1}$ and $\sigma_{2}$ of the underlying Gaussian processes. The parameters $A_{1}, A_{2}, \mu_{1}$ and $\mu_{2}$ of Tab. II, last row, are used. The circle at $\sigma_{1}=0.61$ and $\sigma_{2}=1.00$ marks the result found in the fit of the intensity $I_{W}$.

tally and numerically. The inspection of PDF's of neutral $\mathrm{W}$ density $n_{W}$ and corresponding line emission profiles $I_{W}$ reveals a pronounced signature of double lognormal distributions. This means that "true" temporal averages of $n_{W}$ and $I_{W}$ where background plasma fluctuations are included do not deviate significantly from approximative averages based on mean values for the background plasma as long as the underlying $E \times B$ turbulence does not provide strongly intermittent plasma turbulence. Therefore, in the framework of the theoretical model presented, for typical PSI-2 operation the reconstruction method of the ERO code for comparison with optical spectroscopy is justified and the discrepancies in absolute values of line emission intensity discussed in this work are likely to be caused by other means. Despite these specific findings for the interpretation of PSI-2 experiments the observed double log-normal distribution in the passive scalar model are of interest in the broader framework of Langevin equations including advection. The origin of this particular result will be studied more in detail in future work.

\section{Acknowledgments}

This work has been carried out within the framework of the EUROfusion Consortium and has received fund- ing from the Euratom research and training programme 2014-2018 under grant agreement No 633053. The views and opinions expressed herein do not necessarily reflect those of the European Commission.

\section{References}

[1] A. Kreter, et al., Fusion Sci. Technol. 68 (1), 8-14 (2015). [2] A. Kreter, Fusion Sci. Technol. 59 (1), 51-56 T (2011).

[3] A. Kirschner, et al., Nucl. Fusion 40, 989 (2000).

[4] A. Kirschner, et al., J. Nucl. Mater. 363-365, 91-95 (2007).

[5] E. Marenkov, et al., J. Nucl. Mater. 463, 268-271 (2015).

[6] A. Eksaeva et al., Proc. PSI Conference 2016, Nucl. Mater. Energy, in press

[7] A. Careta et al., Physical Review E 48, 2279-2287 (1993).

[8] J. D. Bao et al., Journal of Computational Physics 197, 241-252 (2004).

[9] D. Reiser, Phys. Plasmas 19, 072317 (2012).

[10] D. Reiser and A. Mekkaoui, Fusion Science and Technology 63,1T, 237-240 (2013).

[11] D. Reiser et al., Phys. Plasmas 21, 032302 (2014).

[12] G. Tynan, C. Holland, J.H. Yu, A. James, D. Nishijima, M. Shimida and N. Taheri, Plasma Phys. Control. Fusion 48, S51 (2006).

[13] N. Kasuya, M. Yagi and K. Itoh, J. Plasma Physics 72, 957 (2006).

[14] N. Kasuya, M. Yagi, M. Azumi, K. Itoh and S.-I. Itoh, J. Phys. Soc. Jpn. 76, 044501 (2007).

[15] V. Naulin, T. Windisch and O. Grulke, Phys. Plasmas 15, 012307 (2008).

[16] P. Popovich, M.V. Umansky, T.A. Carter and B. Friedman, Phys. Plasmas 17, 102107 (2010).

[17] P. Popovich, M.V. Umansky, T.A. Carter and B. Friedman, Phys. Plasmas 17, 122312 (2010).

[18] B.N. Rogers and P. Ricci, Phys. Rev. Lett. 104, 225002 (2010).

[19] M.V. Umansky, P. Popovich, T. A. Carter, B. Friedman and W.M. Nevins, Phys. Plasmas 18, 055709 (2011).

[20] B. Friedman, T.A. Carter, M.V. Umansky, D. Schaffner and B. Dudson, Phys. Plasmas 19, 102307 (2012).

[21] D.A. D'Ippolito, J.R. Myro, S.J. Zweben, Phys. Plasmas 18, 060501 (2011). 